\title{
Picking Up Artifacts: Storyboarding as a Gateway to Reuse
}

\author{
Shahtab Wahid ${ }^{1}$, Stacy M. Branham ${ }^{1}$, Lauren Cairco ${ }^{2}$, D. Scott McCrickard ${ }^{1}$, \\ and Steve Harrison ${ }^{1}$ \\ ${ }^{1}$ Center for Human-Computer Interaction, Virginia Tech, Blacksburg, VA 24060 \\ \{swahid, sbranham, mccricks, srh\}@cs.vt.edu \\ ${ }^{2}$ Department of Computer Science and Quantitative Methods, Winthrop University, \\ Rock Hill, SC 29733 \\ caircoL2@winthrop. edu
}

\begin{abstract}
Storyboarding offers designers the opportunity to illustrate a visual narrative of use. Because designers often refer to past ideas, we argue storyboards can be constructed by reusing shared artifacts. We present a study in which we explore how designers reuse artifacts consisting of images and rationale during storyboard construction. We find images can aid in accessing rationale and that connections among features aid in deciding what to reuse, creating new artifacts, and constructing. Based on requirements derived from our findings, we present a storyboarding tool, PIC-UP, to facilitate artifact sharing and reuse and evaluate its use in an exploratory study. We conclude with remarks on facilitating reuse and future work.
\end{abstract}

Keywords: design reuse, storyboarding, claims.

\section{Introduction}

Storyboards are visual stories of actors engaging in a series of actions for a certain purpose. Typically, they consist of multiple panels made of pictures and an accompanying narrative that illustrates the progression. Used by those involved in the creation of movies, cartoons, and commercials, they are powerful tools in highlighting the most important aspects of a narrative [7],[9]. In Human-Computer Interaction (HCI), storyboards have been used within the design process to illustrate how users may interact with a system [4],[18]. By leveraging graphical descriptions of artifacts, they prove to be powerful tools in pitching and communicating ideas for new technologies.

But what are the artifacts that will be included in the storyboard? Choosing artifacts is key as it is a reflection of the vital functionality that a proposed technology might support. Designers strive to derive new artifacts through creative efforts to improve the design of their products. This involves determining the nature of the artifacts, how they connect to other artifacts, and the visual elements that will represent them.

It is also true that practitioners often informally continue to turn to features that have been used before because they have been proven to stand the test of time. Everyday tools such as remote controls and microwave ovens have maintained the same 
basic design principles for many years. While new iterations continue to be produced, designers naturally reuse techniques of the past-providing impetus behind an often ignored need to gain and even build on what has been used before [24]. Improvements based on prior work can enable designers to potentially continue to maximize successes while avoiding or mitigating identified pitfalls and lowering costs [2]. In fact, the benefits of reuse on usability have been demonstrated [23], justifying efforts within $\mathrm{HCI}$ that have investigated ways of facilitating the reuse of various components often in the form of design knowledge [3],[11],[12],[20]. Although this might seem fruitful, designers exposed to collections of reusable artifacts are often burdened by the large amount and restricted by tools that enforce inflexible design processes, hindering reuse [10].

We propose storyboarding as a fitting activity to provide easier access to design knowledge and aid designers in determining what and how artifacts can be reused. This offers us the chance to combine reusable claims, rationale encapsulating tradeoffs of design features [5], with inspiring images. While pictures can ease access to reusable knowledge, claims serve to familiarize designers with features. Throughout the process of creating the storyboard, identifying and understanding the relationships and connections that exist between components can aid designers in choosing and creating components used in the construction of the final storyboards.

Our goal is to develop a tool that supports storyboarding through reuse. In this effort, we present a study to discover the nature of what the tool must embody. We study groups of designers asked to construct storyboards for a given system by reusing cards consisting of pictures and claims. Results of the study demonstrate that the cards and the relationships identified by designers were useful in jumpstarting efforts to build a prototype through reuse. We then present a tool developed on the results acquired from the study and a preliminary study of its use. We conclude with remarks about lessons we have learned and future efforts.

\section{Related Work}

Storyboarding is the process of describing a user's interaction with a system over time through a series of graphical depictions, often sketches, and units of textual narrative. Storyboards have been used to help understand the flow of the story, to eliminate costly elements of a design, and even to decide how to pitch ideas to others [4],[17]. Thus, they can be seen as early prototypes in the design process. Key aspects of a storyboard are the portrayal of time, the inclusion of people and emotions, the inclusion of text, and the level of detail [21]. Tools such as SILK [13], DENIM [15], and DEMAIS [1] facilitate storyboarding to create prototypes early in design. However, such tools do not support sharing and reusing artifacts.

Design reuse has been researched by many to lower development time and costs [6]. Its value has been seen in industry in different situations. For example, the IDEO Tech Box serves to collect and store objects that can inspire and improve the design of products [4]. Patterns, reusable knowledge structures that incorporate contexts of use, conflicting forces, and potential solutions, have been adopted by those in HCI as a form of design rationale [3] and by those in software engineering [8]. The Yahoo! Patterns Library is an example of a repository that stores reusable components for 
web design [25]. However, such tools lack an overarching design activity, such as storyboarding, that leads to a prototype.

Claims are a way of recording knowledge initially proposed by Carroll and Kellogg [5]. Delivered in informal natural language, claims address a variety of situational and interface aspects that affect the compatibility of the design and user models, such as user satisfaction and feeling of reward, color and object layout, and strength of affordances. They were later proposed as design knowledge units that could be stored in repositories for reuse by designers [20]. A claims library was also created to support their reuse [16]. More recently, they were even included as a part of the design pattern structure by Hughes [11]. However, because claims focus on design rationale, creating tools can be difficult since they often face challenges in capturing, using, and organizing rationale [10].

\section{Uncovering Storyboarding through Reuse}

To investigate how we may provide novice designers access to reusable design knowledge in new ways, we conducted a study in which designers took part in a storyboarding design session. We were particularly interested in how the participants balanced the use of pictures and rationale, connections established among the components, creation of new artifacts, approaches taken to completing the design tasks, and the structures of the storyboards.

\subsection{Participants}

Twenty-one graduate students actively engaged in conducting HCI research or enrolled in a graduate HCI course participated in the study. Their familiarity with claims and storyboarding varied widely, giving us a broad look at the potential ways in which the participants could be impacted by the artifacts.

\subsection{Materials}

A video camera was mounted over a table on which thirty cards describing design features were scattered (see Fig. 1). The front of each card had a picture representing the feature along with a label. The back of the card had a claim for the feature. Blank pieces of paper and pens were also provided, as well as an instruction sheet that explained the task, definitions for concepts such as claims and storyboards, and a prepared design problem.

Each group was given a unique design problem that centered around the need for a notification system. Notification systems are designed to be used in dual-task situations in which a user engaged in a primary task needs to be interrupted for a reason [14]. Some of the design problems we assigned involved notifying nuclear plant operators of changing core temperatures, passengers in airports of flight status changes, commuters of empty parking lot spots while driving, theme park visitors of ride wait times, and students of empty spots for classes they wish to register for. 


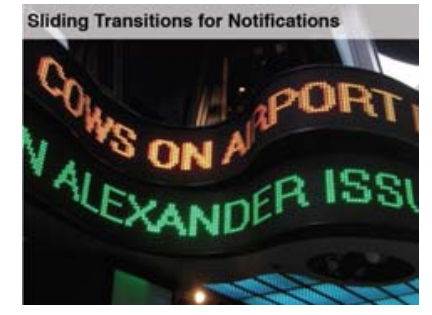

A sliding effect is used to transition from one notification to another

+ Can divert the attention of the user to the notification if needed

+ Can serve as a link between information pieces, allowing users to infer relationships between information pieces

- Constant use of sliding transitions can interrupt the user often

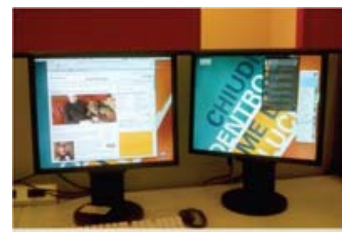

Use of Secondary Displays

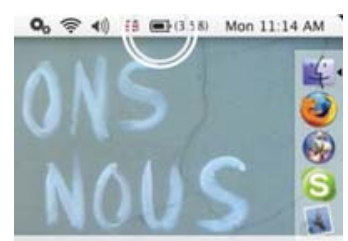

Use of lcons for Notification

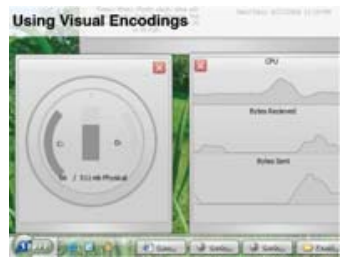

Fig. 1. The front of the cards have pictures illustrating the design feature along with labels (top left). The back describes the consequences of using the feature in the form of a claim (top right). Additional examples of other cards are shown in the bottom row.

\subsection{Procedure}

The participants were randomly divided into 7 groups with 3 in each group. Each group was asked to create a storyboard with 4-7 panels representing a system that would solve the given design problem. They were given the option to create their own cards if they felt they needed a new feature. However, they were told that the new cards must have pictures and claims. If a new card was created, the card was kept in the pile for subsequent groups to see and use. Upon completion of the storyboard, they were asked to write a narrative for the storyboard describing a usage scenario. Each group was told they had 40 minutes, but we did not stop groups that went over the time limit. Once the storyboard was completed, the participants were interviewed as a group for approximately 30 minutes. Interview questions revolved around explaining the constructed storyboard, usage of the cards, and creating cards.

\subsection{Analysis}

We took a grounded theory approach and adopted the open coding technique to analyze the data [19]. Our categories of analysis included analyzing pictures and claims, connections between cards, decision-making, creating cards, task approaches, and storyboard construction. Each group video was analyzed by two coders who watched the complete videos and identified critical points of interest.

\subsection{Results and Discussion}

In this section we present the results focusing on the qualitative data that was acquired from the study. The participants displayed interesting behavior regarding the use of the cards, the connections established between cards, approaches to carrying out the task, and structure of the storyboards. We describe these activities, but expand on only some of them due to space limitations. 
Use of Imagery. For the designers, a critical part of carrying out the activity was familiarizing themselves with the artifacts. Therefore, the participants spent time looking at the cards, sharing them with each other, and referring to them as they talked about possible design ideas. On average, the groups explored cards 86 times. Group 4 explored the cards the most, 120 times, while group 5 investigated cards the least, 56 times. For the majority of the time all the groups kept the cards with the pictures facing up at all times.

The use of pictures to summarize the idea of the reusable artifacts was critical to how the participants explored the cards. The images provided them with ways of recognizing the cards. This was important because of the number of cards that were scattered across the table during the study sessions. One participant from group 1 summarized her thoughts by mentioning the picture, "...was the main thing. I first looked at the photo and then read through." A participant in group 7 emphasized the utility of the pictures: "I guess the picture gives us a very...makes it easier to identify the object so it stands out from the other objects. The pictures are quite different so maybe we employ less effort to recognize the objects instead of going through the text." In response to this comment, a participant in the same group said, "If you had pieces of paper with text on them it would be harder to sort through them." These comments tell us that the use of imagery can potentially make it easier to find and access rationale that would have otherwise been harder to sift through.

The pictures also served to facilitate creative thinking for the design task at hand. For example, one card about showing trends in data had a picture of a graph showing changes in values over time. Group 1 was told to create a system that would allow commuters to find empty parking lot spots. A participant from this group looked at the trends card and said, "you see [some spot] is empty so you click that one and it just opens up...[shows] when it got available and also this graph...you know...gives you an idea about what time of the day it gets full and what time of the day it gets [empty] and stuff like that. That might be a cool thing I think." In this case we notice that feature was directly taken as a possible component for the system being created. There were other cases where an idea was extracted from a picture, but was unrelated to the feature that the picture was representing-perhaps a more creative act. For example, a card about relating preexisting user knowledge to a notification had a picture of a chat window with a chat history. A participant from group 4 picked up the card and said "if you want a timestamp...then [use] something like this." The timestamps happened to be a part of the picture, but were not necessarily there to illustrate the idea of the card. This demonstrates creativity may play a vital role in reuse, potentially making a positive impact on the product. Linking pictures to the claims provided designers with a way of quickly recognizing artifacts, giving them quick access to reusable components that might have otherwise been lost and lowering the burdens of dealing with large amounts of rationale. However, perhaps the more promising utility lies in their ability to inspire, establishing a balance between creative thought and the rationale associated with the images.

Use of Claims. We observed many situations where the participants felt a need to flip the cards over to read the claim for the artifact. On average the groups turned the cards over 20.7 times. Group 4 flipped cards the most, 33 times, and group 1 flipped cards just 10 times. 
The claims served as a way for the participants to familiarize themselves with the details of the feature and the possible consequences of using the feature. Speaking of the claims, a group 4 participant said, "I guess it clarifies what the creator of these [cards] intended to mean by each of these." It served to clarify thoughts if designers were confused about the cards because they misinterpreted or were unsure of what a picture meant. In one instance we saw two participants in group 7 who were looking at a card about personalizing notifications were unsure about what the feature was about. They flipped it over and one participant said, "this seems to be more about the mode of notifications rather than...it's more about the mode rather than the content [of notifications]."

Previous work identified a set of relationships that exist among claims during the design process [22]. This study served to demonstrate that designers, without knowledge of the idea of claim relationships, identify and leverage relationships during their design work once they are familiar with the claim. For example, one common relationship was the idea of generalization vs. specification. Several groups identified a card about notification systems as having this relationship with many of the other existing cards. One person in group 2 mentioned, "[the] notification systems [card] seems too general for this task. It seems it should be the entire overarching second half of our storyboard." In this case, because the group understood the relationship, they were able to decide how relevant the card would be.

Another common relationship that was critical to decision-making involved comparing alternatives. In group 1, a member found two cards on sporadic notification and continuous notification. Having identified through the claims that they are alternatives to each other, the person said, "sporadic vs. continuous...this is something we need to think about. Will it be continuously displayed on your handheld?" A participant in group 3 identified the relationship between voice interaction and touch interaction and then asked, "can we compare the upsides and downsides of the two features to see which one is better?" Comparing alternatives is an important task. Realizing this relationship allows the designers to think about the larger impact reusing the feature could have on the system that is being created.

One advantage of using claims is that it makes designers aware of potential downsides that might need to be mitigated. In such cases, there might be another claim that can serve to reduce or eliminate the negative effects of a claim. This relationship was also realized by a member of group 3. While reading the claim on a card she said, "may miss notification and wait...but we can compensate it with this one. If they miss it they will get a continuous notification like an icon on the corner [of the screen]." Once again, being aware of the tradeoffs and then immediately identifying potential solutions through the connection established paved the way to reusing the card in question.

Finally, two other connections that were observed, but not always explicitly talked about, involved combining cards together and linking information to interaction elements. Often cards were combined together such that both cards would be used in conjunction to represent a new idea. For example, a new notification method could be created by combining a card about a blinking light with an audio notification card. The participants also linked cards appraising the state of an interface to cards about interacting with the elements in the interface. As the participant in group 1 who wanted to use the trends in data card mentioned, the use of the card was also being 
tied to how a user would interact with the feature if it were to be included. These two relationships were used more often in the construction of the storyboards.

As demonstrated through these examples, claims play an important role in the design process. Just as pictures contributed much to the activity, the rationale presented through these claims is often critical to how the participants learn and express ideas to others. When needed, the claims can be used to argue for or against a line of reasoning. The identified connections force designers to think about choosing between claims and how the claims will eventually come together in the storyboard.

Creating New Cards. During the design session we also gave the participants the option to create their own cards if they felt it was needed due to the nature of their design. Because we knew that designers often resist formally contributing knowledge, we wanted to explore how cards were created when needed.

There were only three design sessions, groups 1,4 , and 7 , in which cards were created. In all three cases, the groups had concluded that the new card was essential to the design and therefore needed to be created. To illustrate the process we explain how one group created such a card. Group 1 created a card called using geospatial representation of information so that they could use it for their commuter parking lot system. The conversation that took place during the creation of this card turned out to be quite interesting. First, the person who was writing the claim said, "What was the graphical [card] one? How was that phrased? I lost it...so using geospatial representation of information? I almost feel like...they're related or something." As he proposed a name for the new card, he realized that this card could potentially be similar to a card about graphical information. His need to refer to the other card demonstrated that he wanted to maintain the same level of scope, making it generic and trying not to over-specify the card so that its potential reuse would not be restricted. Thus, his authoring of the claim was influenced by the claims that were already around him. While referring to the graphical information card, he then discussed the possible tradeoffs for the new card: "Yeah, I think the last two [tradeoffs] are particularly relevant. I guess you can specify them for geospatial. If the person doesn't know how the parking lot is laid out... if they see it from overhead...they might understand it the first time they see the map...that sounds like a pretty similar downside..." Finally, once the card was created the participant reflected, "I feel like we're specializing this one...cause it's a kind of graphical information, but it's a special kind. It brings its own pros and cons to an extent in terms of understanding the information. It's about understanding the place, not just the representation of it..." His reflections serve to demonstrate that other cards can influence how new cards are created and that new cards can also be a result of a noted connection. In this case, identifying that relationship with the graphical information card served the group well, allowing them to create a unique and reusable card. This card was eventually reused by group 7 in their own storyboard.

Creating new artifacts is important to reuse because it provides a fresh source of knowledge. While the burdens of creating a new card in terms of content might have been lowered by introducing the picture and the simple structure of claims, we noticed that other factors such as the designer's own knowledge and trust influence whether a card is created. This might be overcome, however, if the designers were exposed to more cards that look like they were contributed by other designers. 
Building Storyboards. While we observed rich interactions as the complete storyboards were constructed, we focus on reporting on the cards in the structures of the storyboards and the strategies used to assemble them. On average the groups had 4.8 panels in their storyboards (where each panel was one or more cards marked by a single section of the written narrative). Group 2 had the most with 6 panels while group 6 had just 3 panels. An average of 2.4 panels contained multiple cards within them. All of the group 5 panels had multiple cards in them. Group 1 and 4 did not have any such panels.

The structures in the storyboards continued to represent the reliance on establishing connections between cards. A common connection was the connection between displaying an artifact and interacting with the artifact, as acknowledged by group 3 participants when they sequenced their cards accordingly. As we mentioned earlier, cards were often combined together to represent a form of fusion. Group 4 was the only group to structure their storyboard such that alternate paths were represented. At one point, their storyboard split into three possible routes, representing different notification methods based on preference, and then converged again. We find it is not only necessary to demonstrate what is being reused, but also how it is being reusedan aspect reflected through the structure of the representation.

We spent time investigating the strategies used throughout the design activity. In general we found the groups typically first familiarized themselves with the cards, made choices, and then constructed the storyboard. Group 3 was the only group that decided to try to group all the cards into categories such as displays, notification methods, and input methods while familiarizing themselves with the cards.

In determining how the group should approach the task, we noticed a clear tension between determining a scenario to inform the design and letting the features determine a possible scenario. Group 1 was the only group that clearly determined a scenario first and then proceeded to create the storyboard. The rest of the groups allowed the combinations of features to determine a possible scenario, but groups 5 and 6 did engage in some initial discussion of a possible task flow. While many groups engaged in a debate about how to approach it, a participant in group 3 put it this way: "So how would you want to start this? Do you want to put individual pieces together and build a storyboard... and then build a story? There are two ways of going about it right? Either you take the abstract chunks and place it then form a storyline or decide [on] a story and then place the chunks." In group 1, we noticed that focusing on the scenario first led to a new card being created because the story was determined independently of what was being offered to them. One should consider whether a scenario-first approach can lead to increased contributions, but we do not have further data to explore this route. While an open and flexible design process can be beneficial, this important tension must be investigated further if we are to work toward proceduralizing storyboarding through reuse as it might have an impact of the quality of the storyboards.

\section{PIC-UP}

Our primary motivation for creating a tool for storyboarding through reuse was to facilitate the collection and sharing of design knowledge. We conducted the study to 
understand how those engaged in storyboarding through reuse would leverage components-giving us a chance to develop a tool that is grounded in actual experiences. The results allowed us to distill a set of goals for a tool that could support this activity:

Supporting recognition and ideation. To avoid having designers expose themselves to large amounts of textual information, images should be used to allow for quick identification. They will also serve as springboards for ideation because they can inspire and provoke thought that can be further solidified with access to rationale.

Discovery through relationships. Because artifacts do not exist in isolation, relationships among them can allow designers to explore other artifacts that might also be relevant. Using relationship types allows designers to find artifacts that are meant to fit the design in a certain way.

Encouraging reusable contributions. While the tool must enable quick and easy creation of artifacts, designers must also be encouraged to make their artifacts as reusable as possible so that others can take advantage of them. Through exposure to other artifacts and guidance, designers can be encouraged to create appropriately scoped and complete artifacts.

Guidance. While an open and flexible process should be adopted, a sense of the important tasks of exploring, deciding, and constructing storyboards should be fostered.

\subsection{PIC-UP Description}

We created a digital storyboarding environment called PIC-UP that builds on the activities carried out for our study and takes advantage of the additional storing, organizing, and guidance that technology enables. Although we focus on the experience on a traditional display, our application was designed to support nontraditional displays such as multiple display environments and tablet PCs.

While PIC-UP can certainly allow designers to create full storyboards from scratch, we focus on supporting creation through reuse. Thus, we provide access to an online library of claims in the form of cards to enable to construction of storyboards by leveraging a table metaphor. Each card appears with a claim title and image and, when necessary, can be flipped to show the complete claim on the back. The cards can be moved easily within the space, allowing users to sift through artifacts as they please.

While our goal was to guide novice designers in their efforts to build storyboards, we did not want to impose a strict process so that designers could be free to carry out the process in their own way. For this reason, we adopted a lightweight method that would illustrate the main types of activities that would need to be carried out. We divided the environment into three resizable spaces: browse, workspace, and storyboard (see Fig. 2). As cards are moved from one space to another, the designer is made aware of the possible tasks that may need to be carried out by referring to a menu on the left of each space.

Browse. The first space in which browsing activities are carried out addresses the need for quick identification of and brainstorming about cards that may be of value to the designer. While initially confronted with a scattered set of cards, users can narrow down and search through the cards in a number of ways with constant focus on images. They can choose to see the top 10 most used cards, a random set of cards, or 


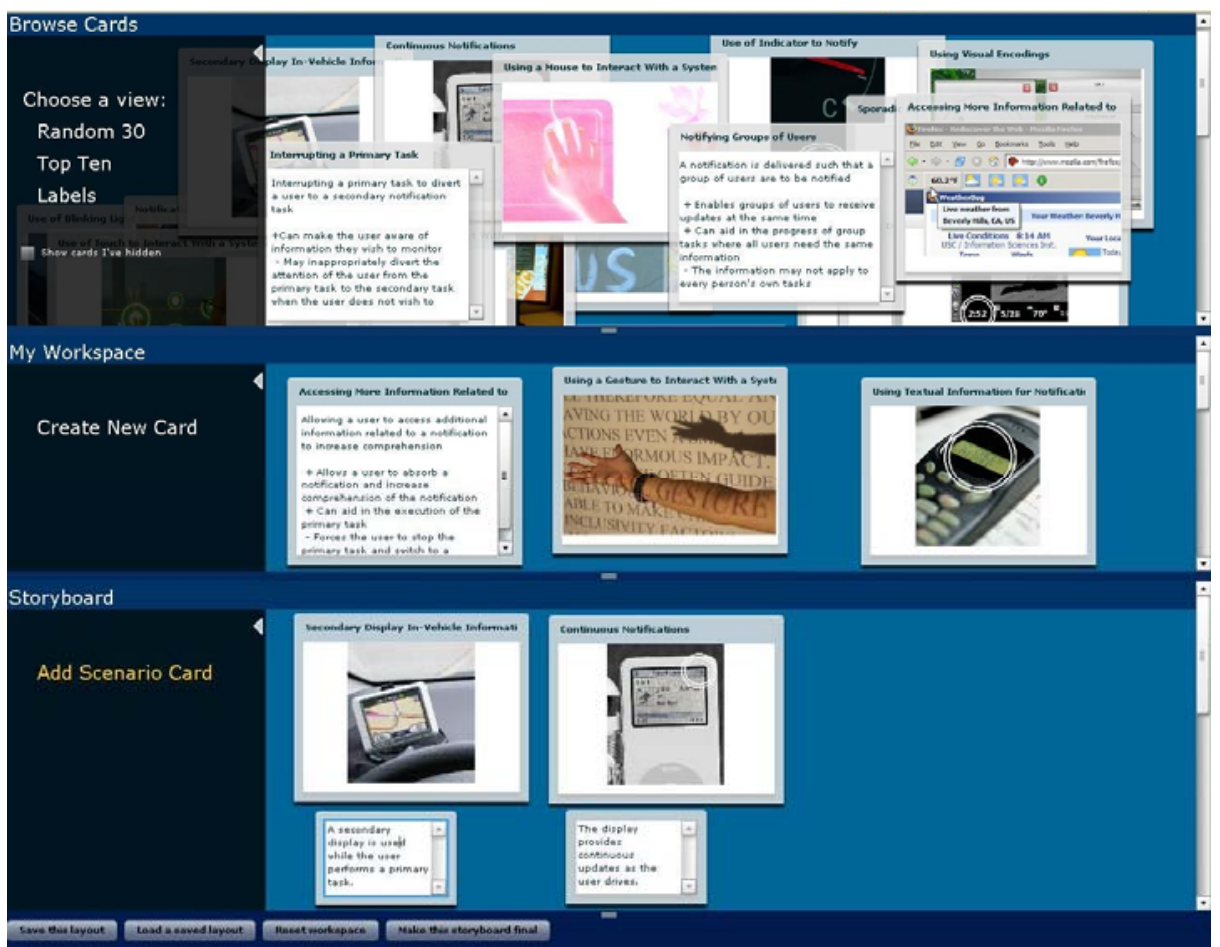

Fig. 2. A screenshot of PIC-UP showing three spaces: browse, workspace, and storyboard. Cards are moved from one space to another as users progress through the design activity.

cards that belong to a certain category through labels assigned to them. Labels such as notification methods, displays, and input methods are used to categorize the cards. Functionality to hide and restore cards is also provided.

A detailed view of the cards allows for further investigation. As we found in the study, the relationships established among the cards can play an important role during the reuse process. This enlarged version of the selected card includes thumbnails of related cards and allows for navigation to them. Relationships such as generalize, specialize, mitigate, fuse, execute, and evaluate are used to describe the connections we observed in our study.

Workspace. Once a card is chosen by a designer it is moved to the workspace. This space is intended to give users a space to collect and decide on cards to use in their storyboard. Users may continue to see the detailed view of cards and read the claims to familiarize themselves with the artifacts.

Because this space is used to collect components, it also gives designers the opportunity to further determine how suitable an artifact might be. In some cases the artifacts might be only somewhat appropriate. An artifact can be edited so that it better fits the context of the system being created. When an artifact is not available, the user may create a new card. Creating a new card involves adding a title, image, and full claim. We deliberately wanted to make the process of creating a new card quick and 
simple to reduce the burden on the designer. When the designer's work is complete, the edited or new cards can be stored for others to view and reuse. Because this creates a source for contributions to the repository, we try to encourage designers to make their contribution as reusable as possible. We believe that exposure to the cards in the browse space can inform designers as to how their own cards should be authored to make them reusable.

Storyboard. The cards that will make it into the final storyboard are moved from the workspace to the storyboard space. The space is used to sequence the cards to represent an appropriate scenario of usage. Our choice of using the table metaphor for our tool proves useful in this space because it continues to allow the designers to represent different paths and relationships in the arrangement of the cards. This section also allows the user to add scenario cards, blank text boxes that the user can type their narrative into and place underneath corresponding artifacts. Like the regular cards, these can be moved around in the same way, although they can only exist within this specific space.

\subsection{Exploratory Study}

To gather feedback on the tool we developed, we conducted an exploratory study with six undergraduate students. The participants previously participated in other sessions of the same storyboarding activity for a different study. Although we realize this gives them an advantage, our primary goal was not to observe their design habits, but to collect initial reactions to aid in further tool refinement to conduct future studies.

Each participant was asked to create a storyboard for a system that would notify a traveler in an airport when their flight was departing. We asked that the storyboard use at least two existing cards, at least one new card, contain three to five panels, and include a narrative describing the usage scenario to ensure they used all of the available functionality. Once the task was complete, participants filled out a survey responding to Likert-scale questions about finding cards and creating cards. They also provided comments on what they liked the most, what they found most frustrating, and any changes or additions they would make to PIC-UP.

We found all participants used between five and eleven cards to construct their storyboards. None chose to edit an existing card for use in their storyboard. We were surprised to find only two of the participants included a picture in the cards they created even though they were free to find an image online or draw an image themselves and import it. This points out that inserting images might be an impediment that could not be overcome easily or was perceived to be time-consuming. While we know there are benefits to having imagery, potential barriers to inserting imagery needs further study as it might be detrimental in the long run.

Bailey et al. found paper and pencil techniques were preferred to communicate design structure by juxtaposing artifacts in a physical space [1]. In our questionnaire, five of six participants reported they would prefer using the digital method for storyboarding over paper-based methods because of the drag-and-drop interface. Perhaps the physical nature of PIC-UP allowed them to juxtapose artifacts in ways that mimicked the findings of Bailey et al. The one participant who preferred paper-based storyboarding mentioned the physical cards would provide more freedom and "responsiveness" and might explain why images were not included in some cards. 
Our questionnaire asked the participants how easy it was to find cards and create new cards on a scale of 1 (very easy) to 5 (very difficult). Participants ranked creating their own cards as easier (mean of 1.7) than finding and reusing preexisting cards (mean of 2.7). No participant ranked the difficulty of either task above 3 , indicating that participants considered both tasks to not be too difficult.

The participants gave us the impression that they generally enjoyed using the tool. When asked what they liked best about the tool, we received positive comments about the emphasis on the visuals, the drag and drop interaction, and the different spaces organizing the work for them. However, they did offer some suggestions for improvements. While viewing images initially helped them find the artifacts they wanted to use, they felt that an arranged positioning of cards (instead of the current scattered placement) would have aided them in finding cards more easily. Others request larger labels for the cards. When creating cards most participants wrote the point and click approach to entering the parts of the card made the task simple, but some participants encountered problems in finding out how to modify their cards.

\section{Conclusions}

Our study on storyboarding demonstrated we can create scenarios of use by reusing artifacts. We found the use of imagery aids ideation, quick recognition, and access to claims that might have otherwise been harder to identify-creating a balance between creativity and design rationale. Key to the building of the storyboard are the relationships that naturally emerge and aid in identifying, deciding on, and sequencing artifacts. Grounded in these observations, we developed a tool to support storyboarding, but also facilitate the artifact creation and sharing needed for reuse. Our research efforts have lead us to learn some important lessons regarding storyboarding and reuse.

Encapsulate reuse within a prototyping activity. Providing reusable artifacts and expecting designers to reuse them is not enough. There is a need to support a design activity that is widely accepted by practitioners. Storyboarding itself is a highly creative and fun activity early in design that can be beneficial to the designers trying to express their ideas and to other stakeholders who wish to understand how a system will function. Since storyboarding naturally has a componentized nature due to the panel structure, it fits well as the basis for a flexible reuse-based design process.

Encourage learning while making contributions. Encapsulating reuse within larger prototyping activities means that contributions are not just being made for the benefit of others, but that designers have a personal reason for creating new artifacts. However, for their contributions to ultimately be useful to others they must be authored in a way that is not too restrictive. Exposure to other artifacts of a similar nature, either through mechanisms such as categories or relationships, can not only familiarize the designers with the domain, but also the ways in which they are created.

Weigh the tradeoffs of structural changes in artifacts. Since we knew of the challenges designers face when trying to reuse, one of our intentions was to explore the benefits of attaching inspiring imagery to claims. While they provide easier access to rationale, it also means that we must explore how to encourage people to also include their own imagery. Although further iterations on the storyboards and support for 
sketches might provide incentives to designers, this teaches us an important lesson in understanding the impact on reuse when trying to shift the balance of the challenges.

Our work provided us with valuable insight into the design concerns that impact the building of storyboards through reuse. Other aspects of the activity, such as the collaboration, can also impact what and how artifacts are reused. We realize there is still more work to be done. We intend to refine PIC-UP to fix identified problems. Additional support for assigning relationships can also be included so that new contributions are not left in isolation in the repository, but connected to the larger network of artifacts. Because our storyboarding is based on generic images as opposed to specific sketches, this places greater emphasis on the narrative when trying to understand the task flow. Further design iterations to specify the storyboard may be required by designers, but we believe this approach can aid novice designers in learning about components needed for their system. Thus, we plan on conducting a summative evaluation on the use of the tool with practitioners to better define how well the storyboards are able to capture the information most desired.

Acknowledgments. This work was partially funded by the National Science Foundation (IIS-0552732).

\section{References}

1. Bailey, B., Konstan, J.A.: Are Informal Tools Better?: Comparing DEMAIS, Pencil and Paper, and Authorware for Early Multimedia Design. In: Conference on Human Factors in Computing Systems (CHI), pp. 313-320 (2003)

2. Bias, R.G., Mayhew, D.J.: Cost-Justifying Usability. Morgan Kaufmann, San Francisco (2005)

3. Borchers, J.O.: A Pattern Approach to Interaction design. In: Conference on Designing Interactive Systems (DIS), pp. 369-378 (2000)

4. Buxton, B.: Sketching User Experiences: Getting the Design Right and the Right Design. Morgan Kaufmann, San Francisco (2007)

5. Carroll, J.M., Kellogg, W.A.: Artifact as theory-nexus: Hermeneutics meets theory-based design. In: Conference on Human Factors in Computing Systems (CHI), pp. 7-14 (1989)

6. Dusink, L., van Katwijk, J.: Reuse Dimensions. In: Symposium on Software Reusability, pp. 137-149 (1995)

7. Finch, C.: The Art of Walt Disney: From Mickey Mouse to the Magic Kingdom. Harry Abrams, New York (1973)

8. Gamma, E., Helm, R., Johnson, R., Vlissides, J.: Design Patterns: elements of reusable object-oriented software. Addison-Wesley Longman, Boston (1995)

9. Hart, J.: The Art of the Storyboard: Storyboarding for Film, TV, and Animation. Focal Press (1998)

10. Horner, J., Atwood, M.E.: Design rationale: rationale and the barriers. In: Nordic Conference on Human-Computer Interaction (NordiCHI), pp. 341-350 (2006)

11. Hughes, M.: A pattern language for user assistance. Interactions 14(1), 27-29 (2007)

12. Landay, J.A., Borriello, G.: Design Patterns for Ubiquitous Computing. Computer 36(8), 93-95 (2003)

13. Landay, J.A., Myers, B.A.: Interactive sketching for the early stages of user interface design. In: Conference on Human Factors in Computing Systems (CHI), pp. 43-50 (1995) 
14. McCrickard, D.S., Chewar, C.M., Somervell, J.P., Ndiwalana, A.: A Model for Notification Systems Evaluation-Assessing User Goals for Multitasking Activity. Transactions on Computer-Human Interaction (TOCHI) 10(4 ), 312-338 (2003)

15. Newman, M., Lin, J., Hong, J., Landay, J.A.: DENIM: an informal web site design tool inspired by observations of practice. Human-Computer Interaction 18(3), 259-324 (2003)

16. Payne, C., Allgood, C.F., Chewar, C.M., Holbrook, C., McCrickard, D.S.: Generalizing Interface Design Knowledge: Lessons Learned from Developing a Claims Library. In: International Conference on Information Reuse and Integration (IRI), pp. 362-369 (2003)

17. Rosson, M.B., Carroll, J.M.: Usability Engineering: Scenario-Based Development of Human Computer Interaction. Morgan Kaufmann Publishers, San Francisco (2002)

18. Sharp, H., Rogers, Y., Preece, J.: Interaction Design: Beyond Human-Computer Interaction. John Wiley \& Sons Ltd., West Sussex (2007)

19. Strauss, A., Corbin, J.: Basics of qualitative research: Grounded theory procedures and techniques. Sage Publications, Inc., Newbury Park (1990)

20. Sutcliffe, A.G., Carroll, J.M.: Designing Claims for Reuse in Interactive Systems Design. International Journal of Human-Computer Studies 50(3), 213-241 (2000)

21. Truong, K.N., Hayes, G.R., Abowd, G.D.: Storyboarding: an empirical determination of best practices and effective guidelines. In: Conference on Designing Interactive Systems (DIS), pp. 12-21 (2006)

22. Wahid, S., Allgood, C.F., Chewar, C.M., McCrickard, D.S.: Entering the Heart of Design: Relationships for Tracing Claims Evolution. In: Conference on Software Engineering and Knowledge Engineering (SEKE), pp. 167-172 (2004)

23. Wania, C.: Examining the Impact of an Information Retrieval Pattern Language on the Design of Information Retrieval Interfaces. Ph.D. Dissertation, Drexel University (2008)

24. Whittaker, S., Terveen, L., Nardi, B.A.: Let's stop pushing the envelope and start addressing it: A reference task agenda for HCI. Human-Computer Interaction 15, 75-106 (2000)

25. Yahoo! Patterns Library, http: / / developer.yahoo.com/ypattern 